\title{
3D Elemental Distributions in Biological Structures by EFTEM Tomography
}

\author{
R.D. Leapman, ${ }^{*}$ G. Zhang, ${ }^{*}$ T.L. Talbot, ${ }^{*}$ V.V. Speransky, ${ }^{*}$ and M.J. Kruhlak** \\ *Division of Bioengineering \& Physical Science, National Institutes of Health, Bethesda, MD 20892 \\ **Experimental Immunology Branch, National Cancer Institute, NIH, Bethesda, MD 20892
}

By combining electron tomography with energy-filtered transmission electron microscopy (EFTEM), it is possible to map elemental composition in three dimensions [1,2]. Previously, we have demonstrated the feasibility of the technique for application to biology by mapping the threedimensional arrangement of ribosomes in unstained plastic sections of eukaryotic cells using tomographic phosphorus imaging to visualize the ribosomal RNA [2]. Those experiments were performed at a beam voltage of $120 \mathrm{kV}$, which limited the useable range of tilt angles to $\pm 55^{\circ}$ for sections of thickness $70 \mathrm{~nm}$. This limitation occurs because plural inelastic scattering distorts the shape of the energy-loss spectrum around the $\mathrm{P} \mathrm{L}_{2,3}$ core-edge as the thickness increases, complicating separation of the background intensity from the signal intensity.

Use of the elemental phosphorus and nitrogen signals has the advantage of providing intrinsic image contrast that reveals the distributions of both nucleic acids and proteins in suitably thin specimens [3]. In the nucleus, $\mathrm{P}$ and $\mathrm{N}$ imaging coupled with correlative light microscopy has the potential to identify large protein-nucleic acid complexes involved in important cellular functions [4]. Such nuclear domains have been difficult to identify using conventional heavy metal staining techniques because of ambiguity in the interpretation of the observed contrast.

To explore whether EFTEM tomography can provide information about the three-dimensional organization of DNA and protein assemblies in the cell nucleus, we have extended the useable specimen thickness up to $100 \mathrm{~nm}$ and collected projected elemental maps over a larger range of tilt angles to decrease the missing wedge in the data collection [5]. This can be achieved by increasing the beam voltage to $300 \mathrm{kV}$, which doubles the inelastic mean free path relative to that at $120 \mathrm{kV}$. Experiments are in progress to acquire dual axis EFTEM tilt series at a beam voltage of $300 \mathrm{kV}$ using an FEI Tecnai TF30 TEM and a Gatan Tridiem imaging filter equipped with a 2048 x 2048 CCD camera that enables nearly single electron sensitivity. Cultured mouse embryo fibroblasts were prepared without osmium fixative and by using Quetol 651 embedding medium. Sections were cut to a thickness of 80 to $100 \mathrm{~nm}$ and mounted on grids without staining. The specimens were coated with a thin $4 \mathrm{~nm}$ layer of evaporated carbon before adsorption of 10-nm fiducial gold particles on both surfaces. Specimens were mounted in a Fischione model 2020 tomography holder. Tomographic reconstructions are computed using the IMOD software from the University of Colorado [6,7].

Images acquired at a specimen tilt angle of $0^{\circ}$ from a region of a fibroblast nucleus below and above the $\mathrm{P} \mathrm{L}_{2,3}$ and $\mathrm{N} \mathrm{K}$ core-edges together with the corresponding ratio maps are shown in Fig. 1a and Fig. 1b. The fiducial gold particles are visible as bright dots in the pre-edge and post-edge images, but this contrast is canceled out in the ratio maps. The $\mathrm{P}$ and $\mathrm{N}$ ratio maps reveal the distributions of DNA and DNA plus protein, respectively. Similar elemental maps acquired from the same nucleus at a specimen tilt angle of $60^{\circ}$ are shown in Fig. 1c and Fig. 1d. Despite the effective doubling of the specimen thickness at the higher tilt angle, reliable $\mathrm{P}$ and $\mathrm{N}$ elemental distributions are achievable at a $300 \mathrm{kV}$ beam voltage. Whereas the bright-field image contrast decreases significantly at higher beam voltages due to the reduced scattering cross section, the inelastic dark-field image contrast actually increases slightly due to reduced plural scattering effects. More importantly, the shape of the energy-loss spectrum changes much more slowly as the specimen is tilted to higher angles.

We are currently applying EFTEM elemental tomography to map the three-dimensional distribution of nuclear DNA and proteins in mouse embryo fibroblasts as well as thymocytes. We are also assessing the extent to which radiation damage limits the attainable spatial resolution. 


\section{References}

[1] P.A. Midgley and M. Weyland, Ultramicroscopy 96 (2003) 413.

[2] R.D. Leapman et al., Ultramicroscopy 100 (2004) 115.

[3] F.P. Ottensmeyer, J. Ultrastruct. Res. 88 (1984) 121.

[4] Y. Ren, M.J. Kruhlak and D.P. Bazett-Jones, J. Histochem. Cytochem. 51 (2003) 605.

[5] A.J. Koster et al., J. Struct. Biol. 120 (1997) 276.

[6] B.J. Marsh et al., Proc. Natl. Acad. Sci. USA 98 (2001) 2399.

[7] D.N. Mastronarde, J. Struct. Biol. 120 (1997) 343.

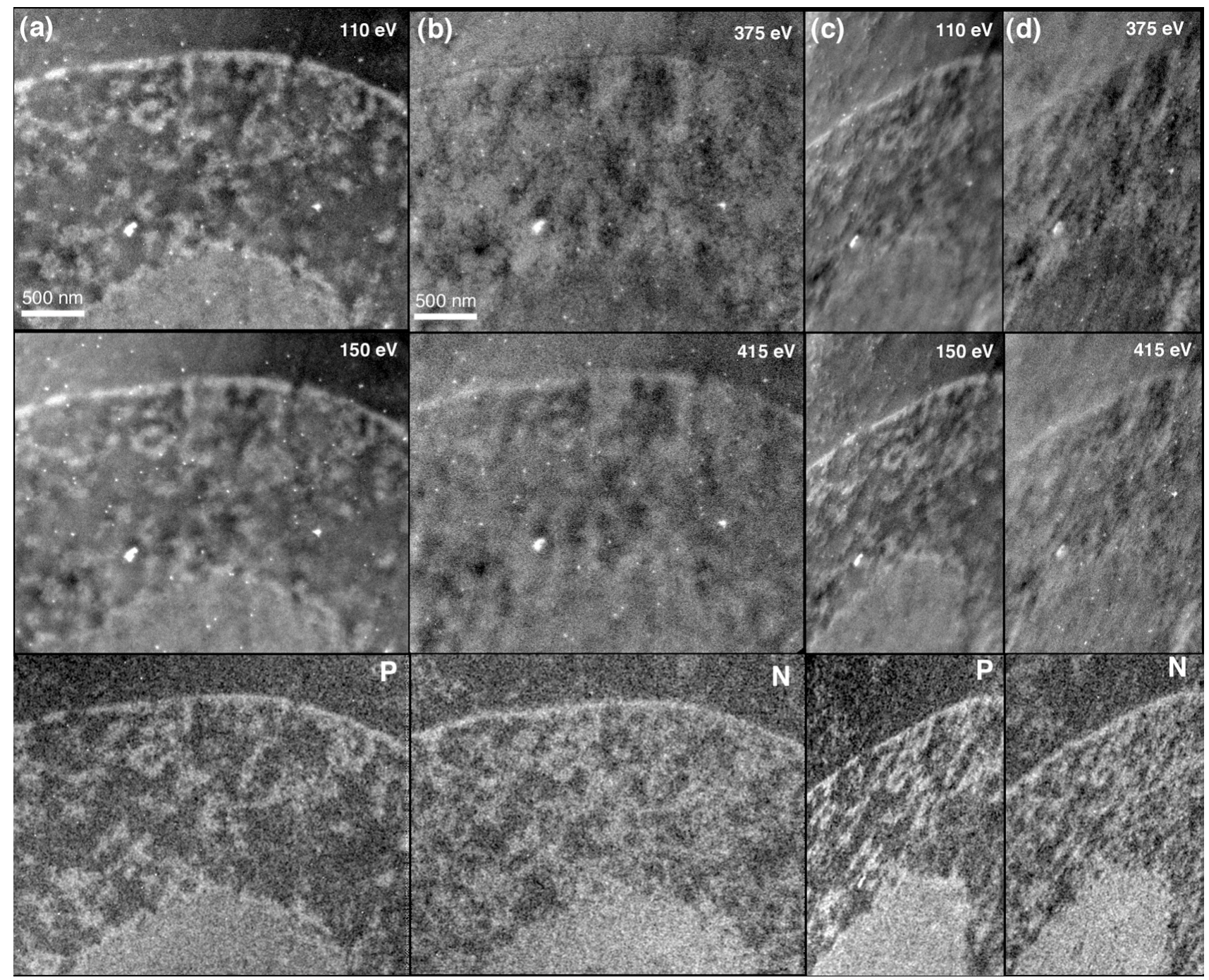

Fig. 1. EFTEM images of fibroblast nucleus in unstained plastic section recorded at a beam voltage of $300 \mathrm{kV}$ : (a) $\mathrm{P} \mathrm{L}_{2,3}$ pre-edge, post-edge and phosphorus ratio map at $0^{\circ}$ tilt; (b) $\mathrm{N} \mathrm{K}$ pre-edge, postedge and nitrogen ratio map at $0^{\circ}$ tilt; (c) $\mathrm{P} \mathrm{L}_{2,3}$ pre-edge, post-edge and phosphorus ratio map at $60^{\circ}$ tilt; (d) N K pre-edge, post-edge and nitrogen ratio map at $60^{\circ}$ tilt. Fiducial gold particles of $10-\mathrm{nm}$ diameter adsorbed onto specimen surfaces are visible as bright dots in both pre-edge and post-edge images due to increased inelastic scattering. These data indicate that EFTEM elemental tomography can be perfomed on 100-nm plastic sections at $300 \mathrm{kV}$ beam voltage. 